\title{
Einige Reaktionen an acetalisierten Ketoestern
}

\section{Doctoral Thesis}

Author(s):

Willimann, Louis

Publication date:

1950

Permanent link:

https://doi.org/10.3929/ethz-a-000090563

Rights / license:

In Copyright - Non-Commercial Use Permitted 


\section{EINIGE REAKTIONEN AN ACETALISIERTEN KETOESTERN und ÜBER EIN APOGERANIOL}

VON DER

EIDGENÖSSISCHEN TECHNISCHEN

HOCHSCHULE IN ZÜ RICH

ZUR ERLANGUNG

DER WÜRDE EINES DOKTORS DER

NATURWISSENSCHAFTEN

GENEHMICTE

PROMOTIONSARBEIT

VORGELEGT VON

LOUIS WILLIMANN

Dipl. Naturwissensdafter

von Gamzil und Luzern

Referent: Herr Prof. Dr. J. Ruzicka

Korreferent: Herr Prof. Dr. PI. A. Plattner

AKADEMISCHE DRUCK-UND VERLAGSANSTALT 


\section{Kapitel I.}

A. Die Reduktion der $\beta$-Ketalester nach B.B. ist von präparativem Interesse. Aus den in guter Ausbeute erhaltenen Ketalalkoholen werden die Ketole in sehr reiner form gewonnen.

Zur Darstellung von $\alpha$ - und $\sigma$-Ketoalkoholen aus den entsprechenden Ketalestern ist die Methode nicht geeignet. Im ersten Fall ist die Ausbeute an Reduktionsprodukt wegen sterischer Hinderung gering, im zweiten treten bei der Ijdrolyse der Ketalalkohole Nebenreaktionen (Bildung von Dihydrofuran) ein. Fermer bereitet die Trennung des Ketoalkohols und des Aethylenglykols Schwierigkeiten. Die $\beta$-Ketole gehen nicht nur in scurem, sondern auch in alhalischem wilieu in $\Delta^{\alpha_{1} /}$-Ketone über. Diese können auch direkt aus àen Ketalalkoholen durch Destillation über Jod gewonnen werden.

B. Dais Aethylenacetal der Acetessigsaiure lässt sich leicht - darstellen. Auch das entsprechende Sturechlorid wir erhalten; doch zersetzt es sich oberhalb $45^{\circ}$.

c. Bei der Einwirkung von Methylmagnesiumjodid auf das Aethylenacetal des d-Butylacetessigesters erhëlt man das entsprecherde tertiäre Ketalcarbinol, das zum freien Ketoalliohol gespalten werden kann.

D. Des Aethylenacetal von Butanol-(I)-on-(3) lässt sich mit Phosphortribromid in Gezenwart von Pyriuin zum k-Bromketal bromieren. Die entsprechende Chlorverbindung ist suf airektem Wege durch ficetalisieren des Chlorketons erhiltlich, vienn wer: beju verfisiren ron Killn Chisroform als Iösungsmittel verwendet. Das $\boldsymbol{C}$-Bronketal reagiert mit kagnesium, die Grignard-Verbindun; ergibt sber mit carbonylverbindungen kein normales 
Umsetzungsprodukt.

E. Des Aethylenacetal des Acetessigesters reagiert mit 0xalester infolge aterischer Hinderung nicht, dasjenige des Ibralinesters glbt dagegen die entsprechende $\alpha$-AethoxaIylverbindung.

7. Bei der Elnwirkung von 0xalester auf Aethylenacetale einfacher Ketone (Aceton, Methyläthylketon, Cyclohexanon) treten unübersichtliche Reaktionen ein. Die entstehenden Produkte konnten nicht identifiziert werden.

\section{KapiteI II.}

Ausgehend von 2-Methyl-5-brom-penten-(2) wurde uber das sekundäre 2-liethyl-octadien-(2,7)-0I-(6) das prinäre 2-MathyIoctadien-(2,6)-ol-(8) hergestellt. Dieser Alkohol unterscheidet sich vom Geraniol mur durch den Mindergehalt der Methylgruppe am $\approx 6$.

Durch Behanalung mit Aluminium-isopropylat in AcetonBenzol geht dieser Alkohol in 2-Methyl-undecatrien-(2,6,8)on-(10) über. Aus diesem "Apo-pseudojonon" liess sich durch Behandlung mit Fhosphorgèure kein cyclisches tzomeres gewinnen. 